\title{
Application of a controlled assembly vocabulary: Modeling a home appliance transfer line
}

\author{
Chase Wentzky ${ }^{2[1]}$, Chelsea Spence ${ }^{1[1]}$, Apurva Patel ${ }^{2[1]}$, Nicole Zero ${ }^{2[1]}$, Adarsh \\ Jeyes ${ }^{1[1]}$, and Alexis Fiore ${ }^{1[1]}$ \\ Dr. Joshua D. Summers ${ }^{2[2]}$, Dr. Mary E. Kurz ${ }^{1[3]}$, and Dr. Kevin M. Taaffe ${ }^{1[2]}$ \\ ${ }^{1}$ Department of Industrial Engineering, Clemson University - Clemson, SC 29634-092 \\ 2 Department of Mechanical Engineering, Clemson University - Clemson, SC 29634-092 \\ ${ }^{[1]}$ Graduate Research Assistant ${ }^{[2]}$ Professor ${ }^{[3]}$ Associate Professor
}

\begin{abstract}
A controlled vocabulary list that was originally developed for the automotive assembly environment was modified for home appliance assembly in this study. After surveying over 700 assembly tasks with the original vocabulary, additions were made to the vocabulary list as necessary. The vocabulary allowed for the transformation of work instructions in approximately $90 \%$ of cases, with the most discrepancies occurring during the inspection phase of the transfer line. The modified vocabulary list was then tested for coder reliability to ensure broad usability and was found to have Cohen's kappa values of $0.671<\kappa<0.848$ between coders and kappa values of $0.731<\kappa<0.875$ within coders over time. Using this analysis, it was demonstrated that this original automotive vocabulary could be applied to the non-automotive context with a high degree of reliability and consistency.
\end{abstract}

Keywords: transfer lines; work instructions; controlled vocabulary; line balancing; standard vocabulary

\section{Why implement a controlled vocabulary?}

Manufacturing and assembly of products is often a collaborative effort between human associates and machines. With an increasing demand in productivity and efficiency from human workers, it is especially important to ensure that the provided work instructions to human workers are accurate and descriptive. This includes both the content and delivery of work instructions. A controlled vocabulary is one such approach within manufacturing which focuses on the content of text-based instructions. It is meant to capture a particular implementation process, to provide clear instructions, and prevent error. In this context, a set of verbs describes the motions performed to do a task, which can then be used to create a standard design for dictating process descriptions [1-3]. Prior to this, written documents describing assembly tasks lacked uniformity and were ambiguous and open to interpretation. This led to a variety of problems ranging from manufacturing defects to safety concerns. To create consistency across technologies and products, controlled vocabulary has been developed, which can help mitigate the 
differences in interpretation. Ford Body and Assembly Operations was one of the first firms to create a language to standardize their work instructions protocol for assembly tasks [1]. From there, they were quickly able to see the benefits, especially with respect to the assembly planners. Not only was the writing of assembly instructions standardized, but ease of machine translation was also apparent. In combination, this helped the planners to predict assembly times based on the verbs used in the work instructions [3]. A controlled vocabulary was also found to be helpful in creating decision support systems and to estimate assembly time [4]. In terms of the Toyota production system, standardization has been found to be one of the bedrocks of kaizen and the continuous improvement benefits of standardization have been well-documented $[5,6]$.

The standardization of vocabulary in instruction manuals or process sheets have shown to provide great value $[1,7]$. As previously mentioned, companies like Ford and Toyota have implemented standard language and found an increase in comprehension by individuals throughout the whole organization. Upon evaluating the standardization, Ford found that clear vocabulary helped limit the number of errors that they encountered in their processes. Additionally, with technology on the rise within manufacturing systems, a standard language allows for not only human understanding, but machine and software understanding as well. In turn, this provides a more cohesive communicative understanding between human and machine. Implementing a standard language helps decrease the complexity of the instructions, which makes the manuals clearer and more concise. As a result, this provides more accurate data on the given tasks and a thorough understanding of the process $[1,6]$.

\section{Controlled Vocabulary Context}

In previous work, a controlled vocabulary was proposed to analyze several aspects of an automotive assembly line [2]. In this paper, this controlled vocabulary is applied to a home appliance transfer line to understand the general applicability of the proposed vocabulary and identify any potential improvements. This standard vocabulary provided in previous work was used, among other things, to better understand the appropriate level of automation for a given assembly task.

Although the aims of the previous study and this current research differ in some respects, the successes of the first study would suggest potential benefits in applying this vocabulary to a home appliance transfer line. This work explored what modifications, if any, would need to be made to apply the automotive standard vocabulary to a home appliance assembly line. Table 1 shows the standard vocabulary used for characterizing assembly tasks in the home appliance assembly line.

Table 1: List of Standard Verbs (adapted from [2])

\begin{tabular}{|l|l|l|l|l|}
\hline Align & Disengage & Lay & Remove & Screw \\
\hline Clamp & Get & Move & Restock & Snap \\
\hline Clean & Insert & Open & Restrict & Tighten \\
\hline Connect & Inspect & Place & Scan & \\
\hline
\end{tabular}


It should be noted that not all of the standardized verbs specified in prior work are listed in Table 1, rather only the verbs used in this case are shown. Additionally, verbs associated with non-value-added tasks are shaded, while the remaining verbs are related to value-added tasks. In this study, value-added tasks are those that transform the product in a way that gives it some desirable feature or trait as defined by the end user.

The manufacturing system currently being evaluated is comprised of transfer lines for the assembly of home appliances. The appliances are assembled through a variety of stamping processes and automated assembly, with roughly 650 employees executing the process. The layout of the plant consists of two separate assembly lines for two different models (P1 and P2) of the appliance. Transfer lines were divided off in a similar fashion, with tasks for Assembly (A), Inspection (I), with several support lines. They will be described in this analysis as $\mathrm{M}_{1}$ and $\mathrm{M}_{2}$, and the three other miscellaneous support lines specified as $\mathrm{X}_{1}, \mathrm{X}_{2}$, and $\mathrm{X}_{3}$.

For the purposes of this analysis, the whole line was broken down into approximately 700 tasks, which were distributed throughout the plant between human assembly, machine assembly, and the infrastructure used to transfer products between workstations. Operators in this facility typically conduct their tasks either sitting or, more commonly, standing; most workers also have some sort of storage space that contain parts or assembly pieces. Some operators use basic hand tools or torque wrenches to aid in their assembly tasks and upon completion of their task, some operators use a foot pedal to advance the assembly to the next workstation.

\section{Application of Controlled Vocabulary}

\subsection{Procedure}

The standard vocabulary identified in prior work [2] was applied to this new assembly process. The tasks used currently on the assembly line were reviewed to apply the controlled vocabulary such that the substantive information within the task list is not modified, but the actions verbs were changed to match the controlled vocabulary. The task list was divided into four equivalent sections which were then processed by four coders. These were then combined to create the complete list of assembly tasks.

The vocabulary used in this analysis is shown below in Table 2, where the first column shows the standard vocabulary from previous work, and the remaining columns show verbs used in task descriptions for the home appliance assembly line. As shown in the table, mapping of verbs in the current task description to controlled vocabulary resulted in a one-to-many mapping. This suggests that assembly instructions currently used in the home appliance assembly line may be using different terms to describe the same action. Additionally, the word "position" in the current task descriptions was found to be mapped to two verbs from the standard vocabulary: "align" and "place." This shows that there is ambiguity in the term "position", and it should possibly be avoided when describing assembly tasks in this setting. 
Table 2. The final proposed standard vocabulary with key.

\begin{tabular}{|c|c|l|l|l|l|l|}
\hline Standard Verb & Value-Add? & \multicolumn{5}{|c|}{ Verb Observed in Original Instruction } \\
\hline Align & VA & Twist & Position $^{1}$ & Turn & Adjust & Organize \\
\hline Clamp & VA & Attach & Clamp & & & \\
\hline Clean & VA & Wipe & & & & \\
\hline Connect & VA & Attach & Install & Hook & Plug-in & Connect \\
\hline Disengage & VA & Unhook & Separate & & & \\
\hline Get & NVA & Obtain & Pick up & & & \\
\hline Insert & VA & Start & Insert & Install & Dip & \\
\hline Inspect & NVA* & Inspect & Check & & & \\
\hline Lay & NVA & Route & Pull & & & \\
\hline Move & NVA & Move & Pull out & Close & Lift & Flip \\
\hline Open & NVA & Unwrap & Open & & & \\
\hline Place & VA & Place & Position ${ }^{2}$ & Insert & & \\
\hline Remove & NVA* & Peel & & & & \\
\hline Restock & NVA* & Restock & Fill & & & \\
\hline Restrict & VA & Secure & Tie & & & \\
\hline Scan & VA & Scan & & & & \\
\hline Screw & VA & Screw & Secure & & & \\
\hline Snap & VA & Snap & & & & \\
\hline Tighten & VA & Tighten & Tool & & & \\
\hline 1- followed by prepositional phrase & & & \\
\hline
\end{tabular}

\subsection{Applicability to Assembly Process}

The process of verb standardization was found to be straightforward for the most part; however, not all of the assembly tasks were successfully converted into standardized verbs. Fig. 1 below shows the result of verb standardization. The graph is divided into seven sections, each of them being a segment or stage of the assembly line. The percentage of verbs that were compatible with the controlled vocabulary are shown by product model (P1 and P2) and by stage ( $\mathrm{A}, \mathrm{I}, \mathrm{M}_{1}, \mathrm{M}_{2}, \mathrm{X}_{1}, \mathrm{X}_{2}$, and $\mathrm{X}_{3}$ ). It should be noted that the " $\mathrm{X}_{3}$ " support line was only present for $\mathrm{P} 1$, and that there was no equivalent support line for $\mathrm{P} 2$.

Overall, $89 \%$ of the verbs in the original work instructions were addressed by the standardization. The " $\mathrm{M}_{2}$ " stage, which corresponds to highly mechanized tasks, had the highest standardization across both product models. Many of the verbs that were not addressed occurred more frequently in stages "I" and "M", which involved irregular tasks. For example, one of the tasks listed in the work instructions was vacuuming excess water from the appliance, with the assembly task described as "Wait time on vacuum." This task was not indicative of the assembly process at hand but was rather an intermediate "irregular" step that related to the assembly environment. 


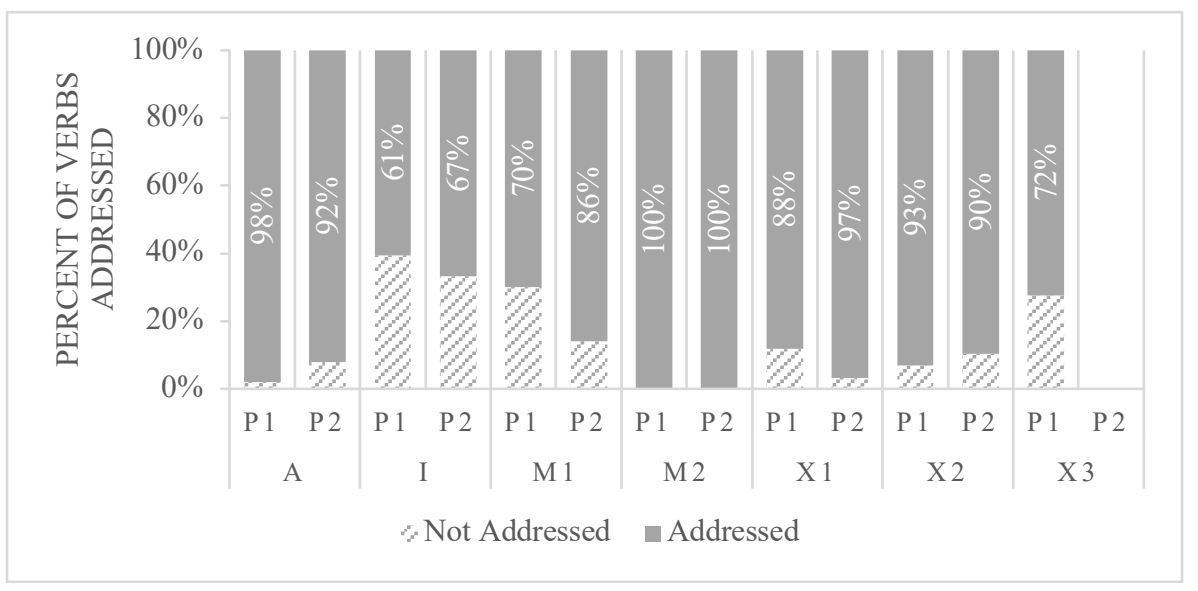

Fig. 1. The results of the verb standardization by model and process stage.

When matching verbs to standardized language, it was important to have precise definitions. For example, place and align are standardized verbs that have similar meanings. To clarify, the group chose to use the verb "align" if the assembly instructions refer to an orientation, distance or angle with respect to another object, otherwise the verb "place" was used. The reason to differentiate between those verbs was to ensure when higher precision in assembly was required. This suggests that in addition to a standard list of verbs, each verb needs to be clearly defined to ensure accuracy and consistency of instructions. An example for each verb may also be beneficial to authors of assembly instructions.

\subsection{Measures of Agreement}

Once the final set of verbs was established, an intercoder reliability analysis was completed within and between coders. The four coders chosen for this test were all graduate students in either mechanical or industrial engineering and had work experience ranging from one to five years. The different coders each applied the standard vocabulary to a random sample of the larger set of work instructions. This sample was gathered by amassing every fifth line of the work instructions provided to assembly associates at their respective workstation. This sampling of the workstation instructions allowed for the entire length of the transfer line to be assessed. Each of the four coders assessed the same sampling of work instructions separately, and each coder's assignment of the standard vocabulary was compared to the other three sets. In order to properly understand the utility of the standard vocabulary amongst individuals with varying backgrounds, Cohen's Kappa $(\kappa)$ was calculated for each of the comparison cases between coders. For intercoder agreement, Kappa values greater than 0.600 are considered to indicate substantial agreement, while Kappa values above 0.800 indicate "almost perfect" agreement $[8,9]$.The Kappa values found between each of the four coders is shown below in Table 3. Cohen's Kappa $(\kappa)$ value for the agreement analysis between the four coders. 
Table 3. Cohen's Kappa (к) value for the agreement analysis between the four coders.

\begin{tabular}{l|cccc} 
& Coder 1 & Coder 2 & Coder 3 & Coder 4 \\
\hline Coder 1 & - & 0.671 & 0.761 & 0.775 \\
Coder 2 & 0.671 & - & 0.848 & 0.715 \\
Coder 3 & 0.761 & 0.848 & - & 0.811 \\
Coder 4 & 0.775 & 0.715 & 0.811 & -
\end{tabular}

Kappa values indicated substantial agreement amongst the four coders, which is unsurprising given that all four had been given training with the standard vocabulary key in Table 2. To further analyze the utility of the new standard vocabulary, a similar analysis was completed using Cohen's Kappa, but in this instance, the analysis was within each of the four coders. After five weeks, the original four coders were given the same set of randomly selected tasks from the broader list of work instructions. Without consulting their previous responses, the coders were asked to apply the standard vocabulary once again, and agreement was assessed between this set of responses and their first set of earlier responses. This application of Cohen's Kappa helps to ensure that the vocabulary is a useful tool over time, and that the original intercoder agreement was not simply the result of recent training but was indicative of the vocabulary's usability. The Kappa values for each of the raters was greater than 0.731 , suggesting a high-level of internal consistency amongst coders using the standard vocabulary [8,9]. In summary, the "rating" or application of controlled vocabulary in this case was found to be consistent between coders and within coders, suggesting that the underlying method of vocabulary application is robust.

Because each of the four coders was trained using the standard verb key, it may seem odd that there was not complete agreement $(\kappa=1)$ amongst the coders. However, there were a few discrepancies present in the sample of work instructions that could explain this variation. Namely, there were discrepancies in the number of tasks that an individual work instruction represented. Some work instructions explicitly stated two distinct tasks (e.g. "Open door and insert weight.") or instructed the operator to interact with multiple parts (e.g. "Get bolts (3)."). In these cases, if coders split those tasks into several different tasks, the test was adjusted to only include verb assignments that each coder made. However, work instructions that included implied conditions may also affect how the coder assigned a standard verb based on prior knowledge of the process or the parts and tools being used. This ambiguity was also another possible explanation for some of the variation among coders in the sample tasks.

\section{$4 \quad$ Summary}

Due to the successes observed in the automotive industry from using standard vocabulary, an existing assembly vocabulary was modified to evaluate a home-appliance manufacturing line. Nearly $90 \%$ of the tasks listed in the original work instructions were addressed within the standardization. In conclusion, the use of standard vocabulary stemming from the automotive industry applied well to the home-appliance system 
with only slight variance. To test the agreement of the standard vocabulary list, an intercoder reliability test was conducted between and within the coders. This showed the strength of long-term agreement within the coders individually and the agreement between all the coders together. As a result, it holds true that controlled vocabulary is useful over time and the application was robust. Several additional benefits have been observed during the application of the standard vocabulary including:

- automation of assembly time estimation $[10,11]$

- benefits for system modelling using discrete event simulation [12]

- identifying the value stream of a product

- the elimination of potential translation errors

These additional benefits will be expounded upon in a later work, but also serve to demonstrate the optimized process flow that may accompany the implementation of a standard vocabulary in manufacturing and assembly.

\subsection{Limitations}

During the process of applying the standard vocabulary list to such a large number of assembly tasks, several limitations became apparent. Application of the standard vocabulary was done through document review only, meaning the actual work being done on the assembly was not always observed in order to apply the vocabulary onto existing instructions. While this is one of the biggest advantages of applying the vocabulary, this also presented some challenges. Even when certain verbs were straightforward to standardize, there were some exceptions when the task description was vague. The verb "obtain" has a specific meaning making it simple to assign a descriptive and accurate verb, while other verbs, like "secure", left more room for interpretation (i.e. a part could be secured by screwing, tightening, or clamping). When these verbs were encountered, more work was required to find the most accurate standardized language. By looking at previous tasks, more context could be gained. If an operator needs to get screws immediately before the part is secured, there is a high likelihood that the part is screwed in. Using the pictures that accompany the work instructions can also help clarify exactly what the operator is doing. If none of the verbs on the standardized list were applicable, new ones could be added.

\subsection{Future Work}

Future work would benefit from looking at the physical work done by the operators and seeing the value of applying the modified controlled vocabulary. Additionally, future work can also determine whether an appropriate level of specificity is provided within the standard vocabulary, and whether the level of specificity needed is different based on the products being assembled. Also, using the standard vocabulary for other manufacturing settings can help expand the applicability of the automotive assembly standard vocabulary from [2]. Because this application was successful for the homeappliance manufacturing line, it is expected that applying the standard vocabulary in other manufacturing systems could also produce positive benefits. 


\section{References}

[1] Rychtyckyj, N., 2006, "Standard Language at Ford Motor Company: A Case Study in Controlled Language Development and Deployment," Cambridge, Massachussets.

[2] Salmi, A., David, P., Blanco, E., and Summers, J. D., 2016, "Standardized Vocabularies for Assembly Systems Modelling and Automation Alternatives Description," Vol. 1B 36th Comput. Inf. Eng. Conf., p. V01BT02A009.

[3] Peterson, M., Mocko, G. M., and Summers, J. D., 2013, "Knowledge Management for Semi-Automated Automotive Assembly Instruction Authorship and Translation," ASME International Design Engineering Technical Conferences and Computers and Information in Engineering Conference, ASME, Portland, OR, pp. DETC2013-13070.

[4] Miller, M., Griese, D., Peterson, M., Summers, J. D., and Mocko, G. M., 2012, "Reasoning: Installation Process Step Instructions as an Automated Assembly Time Estimation Tool," Proceedings of the ASME Design Engineering Technical Conference, ASME, Chicago, IL, pp. DETC2012-70109.

[5] Johansson, P. E. C., Lezama, T., Malmsköld, L., Sjögren, B., and Ahlström, L. M., 2013, "Current State of Standardized Work in Automotive Industry in Sweden," Procedia CIRP, 7, pp. 151-156.

[6] Pereira, A., Abreu, M. F., Silva, D., Alves, A. C., Oliveira, J. A., Lopes, I., and Figueiredo, M. C., 2016, "Reconfigurable Standardized Work in a Lean Company--a Case Study," Procedia CIRP, 52, pp. 239-244.

[7] Peterson, M., 2012, "Standardization of Process Sheet Information to Support Automated Translation of Assembly Instructions and Product-Process Coupling."

[8] McHugh, M. L., 2012, "Interrater Reliability: The Kappa Statistic," Biochem. medica Biochem. medica, 22(3), pp. 276-282.

[9] Landis, J. R., and Koch, G. G., 1977, "The Measurement of Observer Agreement for Categorical Data," Biometrics, pp. 159-174.

[10] Miller, M. G., Summers, J. D., Mathieson, J. L., and Mocko, G. M., 2014, "Manufacturing Assembly Time Estimation Using Structural Complexity Metric Trained Artificial Neural Networks," J. Comput. Inf. Sci. Eng., 14(1).

[11] Cakmakci, M., and Karasu, M. K., 2007, "Set-up Time Reduction Process and Integrated Predetermined Time System MTM-UAS: A Study of Application in a Large Size Company of Automobile Industry," Int. J. Adv. Manuf. Technol., 33(3), pp. 334-344.

[12] Lee, Y.-T. T., Riddick, F. H., and Johansson, B. J. I., 2011, "Core Manufacturing Simulation Data--a Manufacturing Simulation Integration Standard: Overview and Case Studies," Int. J. Comput. Integr. Manuf., 24(8), pp. 689-709. 